\title{
Changes in the aerobic faecal flora of patients treated with antibiotics for acute intra- abdominal infection
}

\author{
Annika Samuelsson, Barbro Isaksson, Abbas Chabok, Jon Jonasson, \\ Lennart E Nilsson, Olle Eriksson and Håkan Hanberger
}

\section{Linköping University Post Print}

N.B.: When citing this work, cite the original article.

Original Publication:

Annika Samuelsson, Barbro Isaksson, Abbas Chabok, Jon Jonasson, Lennart E Nilsson, Olle Eriksson and Håkan Hanberger, Changes in the aerobic faecal flora of patients treated with antibiotics for acute intra-abdominal infection, 2012, Scandinavian Journal of Infectious Diseases, (44), 11, 820-827.

http://dx.doi.org/10.3109/00365548.2012.695455

Copyright: Informa Healthcare

http://informahealthcare.com/

Postprint available at: Linköping University Electronic Press http://urn.kb.se/resolve?urn=urn:nbn:se:liu:diva-86555 
Changes in the aerobic faecal flora of patients treated with antibiotics for acute intraabdominal infection.

Annika Samuelsson ${ }^{1.2}$, Barbro Isaksson ${ }^{1.2}$, Jon Jonasson ${ }^{3}$, Lennart E Nilsson ${ }^{2}$, Olle

Eriksson $^{4}$, Håkan Hanberger ${ }^{5}$, Abbas Chabok $^{6}$

From the ${ }^{1}$ Department of Infection Control, University Hospital Linköping, and 2Department of Clinical Microbiology, and ${ }^{3}$ Department of Clinical Genetics and ${ }^{5}$ Department of Infectious Diseases at the Department of Clinical and Experimental Medicine, Faculty of Health Sciences Linköping University and the ${ }^{4}$ Department of Computer and Information Science, Linköping University, Linköping Sweden, ${ }^{6}$ Colorectal Unit, Department of Surgery, and Centre for Clinical Reserch Uppsala University, Västmanlands Hospital, Västerås, Sweden

Keywords: antibiotic resistance, faecal flora, intra-abdominal infection.

Running headline: Changes in the aerobic faecal flora

Corresponding author: A. Samuelsson, Department of Infection Control, University Hospital, SE-581 85 Linköping, Sweden.

Phone: +46101039622

Fax: +46101031165

E-mail: annika.samuelsson@lio.se

Declaration of interests: this work was supported by the Investigator Initiated Study Programme, MSD, and the County Council of Östergötland. 


\section{Abstract}

Background: An open observational study was performed to investigate changes in the rectal flora and antibiotic susceptibility among faecal bacteria in patients treated with antibiotics for acute intra-abdominal infection.

Methods: One hundred and forty patients with acute intra-abdominal infection requiring antibiotic treatment and hospitalisation were included. Eight surgical units from the southern part of Sweden participated, between January 2006 and November 2007. Antibiotic treatments were according to local guidelines. Rectal swabs were obtained on admission (Sample 1) and 2-14 days after the end of antibiotic treatment (Sample 2). Aerobic bacteria and yeasts were analysed. The material was divided into two groups; one group with Enterobacteriaceae and one group with non-fermentative gram-negative bacteria. The susceptibility to antibiotics in each group was compared between Samples 1 and 2. Results: The main finding of this study on patients with severe intra-abdominal infections was a shift following antibiotic treatment in the aerobic faecal flora from Escherichia coli to other more resistant Enterobacteriaceae, Enterococcus faecium and yeasts. The susceptibility to cephalosporins and piperacillin-tazobactam decreased in Enterobacteriaceae.

Conclusion: Following antibiotic treatment, a shift in the aerobic rectal flora to species with intrinsic antibiotic resistance was observed; indicating that emergence of resistance is not due to new mutations but rather to selection of more resistant species. This should be taken into account when designing treatment for secondary intra-abdominal infections.

\section{Introduction}


The intestinal tract is an important bacterial reservoir and source for transmission of hospitalacquired infections.[1]. The intestinal tract contains 300-500 different species of bacteria that are most abundant in the large intestine and rectum. The faecal mass contains up to $10^{11-} 10^{12}$ living bacteria per gram and about $60 \%$ of its weight consists of bacteria [2-4]. This provides a complex and dynamic ecosystem of living bacteria, which contributes to energy harvest from food, and plays an important role in the maturation of the human immune system. Intra-abdominal infections are often of poly-microbial origin were Escherichia coli and other species of Enterobacteriaceae dominates followed by enterococci $[5,6]$. Other important pathogens are staphylococci, yeasts and anaerobic bacteria. When a patient is treated with systemic antibiotics the resident bowel flora will also be unduly influenced. Broad-spectrum antibiotic treatment disturbs the balance between different bacterial species in the bowel and increases the incidence of antibiotic resistant Enterobacteriaceae [7, 8]. The aim of the present open observational study was to investigate changes in the rectal flora and the emergence of antibiotic resistance in Enterobacteriaceae and non-fermentative gramnegative bacteria, among patients with intra-abdominal infection receiving antibiotics.

\section{Materials and methods}

\section{Study population}

Inclusion criteria were adult patient, age over eighteen years, admitted to a surgical ward having a suspected diagnosis of complicated intra-abdominal infections requiring surgical intervention with the following criteria: 1) Cholecystitis (including gangrenous) and either rupture, perforation or progression of the infection beyond the gallbladder wall. Surgical intervention or CT/Ultrasound confirmed diagnosis. 2) Diverticular perforation/abscess requiring surgical intervention or CT/ultrasound confirmed abscess without surgical intervention. 3) Perforation of appendix with local purulent fluid if surgical intervention is performed or abscess confirmed with CT/Ultrasound without surgical intervention. 4) Acute gastric and duodenal perforations, only if operated on $>24$ hours after perforation occurs. 5) Traumatic perforation of the intestines, only if operated on $>12$ hours after perforation occurs. 
6) Peritonitis due to perforated viscus, or other focus of infection (but not spontaneous bacterial peritonitis associated with cirrhosis and chronic ascites). 7) Intra-abdominal abscess including of liver and spleen abscesses. Alternatively patients with suspicion of any above-mentioned diagnoses were the surgeons chose non-surgical treatment with antibiotic treatment with or without percutaneous intra-abdominal drainage. In the later group we intended to include patients with at least 5 days of antibiotic treatment if there was no surgical intervention, but since there was an intension to treat design we also decided to include patients with shorter treatment.

Participating units were surgical units in eight hospitals from the mid and southern parts of Sweden (Norrköping, Västerås, Göteborg, Örebro, Helsingborg, Uppsala, Linköping and Karlstad) participated in the study between January 2006 and November 2007. Informed consent was obtained from each patient and the study was approved by the Local Ethics Committee at Linköping University Hospital and followed the Helsinki declaration of 1975. Data registered for the study included age, gender, inclusion diagnosis, surgical interventions, discharge diagnosis, co-morbidity (malignancy, chronic obstructive pulmonary disease, and diabetes mellitus), previous antibiotics and travel outside Sweden during the last 6 months were recorded. Antibiotic treatments were introduced according to local guidelines and traditions, and were chosen by the attending physician,

\section{Bacterial and yeast cultures}

To get samples before antibiotic treatment of a patient with an acute need of antibiotic treatment we had to choose rectal swabs in favour of faecal samples of practical reasons and there was not time waiting for defecation before antibiotic treatment. The sample after antibiotic treatment was obtained in the same way as the pre-exposure sample. Rectal 
swabs were obtained on admission (Sample 1) and 2-14 days after the end of antibiotic treatment (Sample 2). Rayon swabs with transport medium (Copan, Brescia, Italy) were used. All samples were sent to the Clinical Microbiology Research Laboratory, Linköping University. At the laboratory the swabs was directly put down into $1.0 \mathrm{ml}$ of freeze broth (Nutrient broth, No 2, Lab m Bury, United Kingdom, containing glycerol 15\%) in a sterile glass tube and shaken thoroughly. The freeze broth was transferred to a plastic storage tube. The rayon swab tip was cut off directly down into the freeze broth in the storage tube and stored at $-70^{\circ} \mathrm{C}$ until analysis. When analysing the samples, they were thawed and once again shaken. The original rayon swab was handled with sterile technique and stroked with a primary streak on one chromogenic UTI-agar plate (Oxoid, Basingstoke, United Kingdom) and stroked over a second UTI-agar plate using a dish rotator (Bio-Merieux, Marcy l'Etoile, France). Chromogenic UTI medium (Oxoid, Basingstoke, United Kingdom) is a chromogenic agar plate that eases the identification of uropahtogenes like Enterobacteriaceae, enterococci and staphylococci by different coloration of colonies useful in mixed cultures [9]. To facilitate identification of different species, antibiotic discs (ampicillin aztreonam, ceftazidime, cefotaxime and linezolid; Oxoid, Basingstoke, United Kingdom) were placed on one of the plates. The agar plates were incubated overnight at $35^{\circ} \mathrm{C}$. Different bacterial colonies were identified visually and bacterial isolates considered to be different species or subspecies found, were identified to species or family level using standard biochemical methods [10].

$25 \mu \mathrm{L}$ of the freeze medium were cultured in Yeast-nitrogen-base- broth (BD, Franklin Lakes, New Jersey, USA) with gentamicin 50mg/L and chloramphenicol $50 \mathrm{mg} / \mathrm{L}$ (YNB-broth) and incubated at $35^{\circ} \mathrm{C}$ until growth or for a maximum of five days. The YNB-broth was streaked over a Sabouraud-agar plate (Oxoid Limited, Hampshire, United Kingdom) with chloramphenicol $50 \mathrm{mg} / \mathrm{L}$ and gentamicin $50 \mathrm{mg} / \mathrm{L}$ and cultured two days at $35^{\circ} \mathrm{C}$. Colonies growing on the plate were visually inspected and classified as Yeasts after gram staining and microscopy. 


\section{Antibiotic susceptibility testing}

Antibiotic susceptibility testing was performed by disc diffusion according to methods and breakpoints according to guidelines of the Swedish Reference Group for Antibiotics (SRGA) (http:// www.srga.org 2007-2008). The isolates were classified as sensitive (S), intermediate (I) or resistant (R). Isolates defined as I or R showed decreased susceptibility. All aerobic gram-negative rods were tested with imipenem, ceftazidime, ciprofloxacin, gentamicin and piperacillin-tazobactam. Enterobacteriaceae were further tested with cefuroxime,cefotaxime and trimethoprim-sulphamethoxazole

The dominant bacterial population and also subpopulations of Enterobacteriaceae and nonfermentative gram-negative rods were tested for antibiotic susceptibility. Each species was counted once only per patient and sample when measuring distribution of species. In order to detect emergence of resistance, we analysed more than one isolate per species from each patient. We measured the occurrence of decreased susceptibility in all of the isolates for each species and patient.

\section{Detection of extended-spectrum-betalactamase (ESBL)}

Isolates of Enterobacteriaceae with decreased susceptibility to cefotaxime or/and ceftazidime were suspected producers of ESBL. Phenotypic double-strip Etest (AB Biodisk, Solna, Sweden) with cefotaxime with and without clavulanic acid, ceftazidime with and without clavulanic acid or cefepime with and without clavulanic acid were used to confirm classical ESBL-enzymes such as CTX-M, TEM and SHV designated ESBL $L_{A}$. Etest with cefotetan with or without cloxacillin were used to confirm plasmid $\mathrm{AmpC}$, designated $\mathrm{ESBL}_{\mathrm{M}}$. When the minimum inhibitory concentration (MIC) ratio of cephalosporin/cephalosporin with clavulanic acid $\geq 8$ or a phantom/deformation zone occurred, an ESBL-fenotype was considered. When the minimum inhibitory concentration (MIC) ratio of cefotetan/cefotetan with cloxacillin $\geq 8$ or a 
phantom/deformation zone occurred, an AmpCL-fenotype was considered. Klebsiella pneumoniae isolates with phenotypic AmpC were considered to have a plasmid AmpC genotype. Isolates with $\mathrm{ESBL}_{\mathrm{A}}$-phenotype were genetically confirmed using PCR as described earlier [11] to detect enzymes of CTX-M, and SHV and TEM genotype. E. coli isolates with AmpC phenotype were genetically confirmed by multiplex PCR according to Perez and Hanson [12] to verify plasmid mediated AmpC.

\section{Statistical analysis}

In the colonisation analysis the material was summarised as indicator variables. The indicator variables were presence or absence of each particular species; quantity was not taken into consideration. The gain or loss of a bacterial species was compared between Samples 1 and 2 using McNemars test. The total number of species present was tested with pair-wise student's t-test.

The fact that all species were not found in each sample made it difficult to evaluate emergence of resistance in each species, hence we divided the material into a group with Enterobacteriaceae (EBC) and non-fermentative gram negative bacteria (NF).

The EBC group included all Enterobacteriaceae species and the NF group included P aeruginosa, Pseudomonas spp (Pseudomonas non $\mathrm{P}$ aeruginosa), and Acinetobacter spp. Each combination of antibiotic and group were analysed separately. The fraction of Enterobacteriaceae with decreased susceptibility in Samples 1 and 2 from each patient was calculated. The fraction of non-fermentative with decreased susceptibility in Samples 1 and 2 was calculated for each patient. The fraction of decreased susceptibility was defined as the number of species with decreased susceptibility divided by the number of species found. Finally the mean fractions of species with decreased susceptibility in the study population were compared between Samples 1 and 2 with two-way analysis of variance with time as fixed factor and patient as random factor.

$P$ values $<0.05$ were considered significant. 


\section{Results}

\section{Population}

One hundred ninety seven patients were initially included. Fifty five patients were subsequently excluded due to failure of sampling, and 2 patients were excluded due to missing demographic data. Thus, samples from 140 patients (70 women and 70 men) were investigated in the final analyses. Surgery was performed in 83 patients (59.2\%) the most common procedure being appendectomy $(44 ; 31.4 \%)$ followed by colectomy and cholecystectomy (13; $9.3 \%$ respectively). Fifty seven (40.7\%) patients were not operated upon.

Eighty two (59\%) patients had a concomitant diagnosis. Diagnoses were dominated by hypertension 34 patients (24\%), followed by diabetes 11 patients (8\%), malignancy 6 patients (4\%), asthma 6 patients (4\%), chronic arthritic disease 5 patients (4\%) and chronic obstructive pulmonary disease 4 patients (3\%). Twenty seven patients (19\%) had received antibiotic treatment within six months preceding inclusion, and 34 patients (24\%) had travelled outside Sweden. Data was missing for 8 patients regarding previous travel and antibiotic treatment. (Table I).

\section{Antibiotic treatment}

Treatments in the carbapenem group included imipenem, meropenem and ertapenem. Correspondingly, cefotaxime, cefuroxime, ceftibuten and cefadroxil were used in the 
cephalosporin group. The quinolone group included levofloxacin, moxifloxacin and ciprofloxacin.

Ninety- five of 140 patients (67.9\%) received a combination of antibiotics with frequent change of antibiotics. Uniform treatment was defined as receiving only antibiotics from only one antibiotic class or one combination of antibiotics the whole treatment episode. The median length of antibiotic treatment was 10 days with a range of 1 to 95 days. Of all patients, $8(5.7 \%)$ received a carbapenem, 8 (5.7\%) piperacillin-tazobactam, $23(16.4 \%)$ received a combination of cephalosporin and metronidazol, $4(2.9 \%)$ received fluoroquinolne treatment as a single treatment or in combination with metronidazole, 1 patient $(0.7 \%)$ each received a cephalosporin or a combination of cephalosporin and aminoglycoside as a uniform treatment. Fig 1.

\section{Microorganisms isolated}

The three most common aerobic bacteria isolated in Sample 1 were $\mathrm{E}$ coli $(90.0 \%$ of all patients) followed by E. faecalis (57.9\%) and yeast (31.7\%). In Sample 2 yeast was the most common isolate $(70.7 \%$ of the patients), followed by $E$ faecalis $(64.3 \%)$ and $E$ coli $(57.9 \%$.) Other differences observed between Samples 1 and 2 were a decrease in $\mathrm{P}$ aeruginosa (16.4\% in Sample 1 and 5.7\% in Sample 2), an increase in E faecium (21.4 and 50.0\%, respectively) and Enterobacteriaceae not further typed (3.6 and 10.7\%, respectively). (Table II)

There was thus, a significant loss of $E$ coli and $P$ aeruginosa and a significant gain in $E$ faecium and yeasts between Samples 1 and 2. The group of Enterobacteriaceae, which were not identified further with routine laboratory tests also gained in numbers. (Figure 2)

\section{Antibiotic resistance}


The mean fraction of Enterobacteriaceae with decreased susceptibility to cefotaxime per patient, increased from 0.357 before antibiotic treatment to 0.507 after antibiotic treatment. The corresponding figures for cefuroxime and piperacillin-tazobactam were an increase from 0.600 to 0.800 and from 0.307 to 0.450 respectively. There were no differences in the mean fraction of Enterobacteriacae with decreased susceptibility to ciprofloxacin, gentamicin, imipenem and trimethoprim-sulphamethoxazole, per patient, before and after antibiotic treatment.

In the group of non-fermentative gram-negative rods the mean fraction of decreased susceptibility to antibiotic, per patient, decreased for imipenem from 0.043 to 0.007 . In this group there were no significant differences in antibiotic susceptibility per patient, before and after antibiotic treatment, to ceftazidime and ciprofloxacin. (Table III)

Two (1.4\%) patients had ESBL-producing E coli in Samples 1 and 2. One (0.7\%) patient had ESBL-producing E coli in Sample 1 only and one (0.7\%) patient in Sample 2 only. ESBLenzymes found were CTX-M 15/28, CTX-M 14 analogous and CTX-M 3/22. Other enzymes found were TEM-1, TEM-2 and SHV-1 alike. Four (2.8\%) patients had Klebsiella pneumoniae isolates with plasmid Amp-C before antibiotic treatment and two (1.4\%) still had these after antibiotic treatment. No other ESBL-producing Enterobacteriaceae was found.

\section{Discussion}

The main finding of this study on patients with severe intra-abdominal infections was a shift in faecal floral pattern from E.coli to other Enterobacteriacae, E.faecium and yeasts following antibiotic treatment. The fraction of decreased susceptibility to cephalosporins and piperacillin-tazobactam in Enterobacteriaceae increased after antibiotic treatment. The loss of $E$ coli and gain of other Enterobacteriaceae, the shift from $E$ faecalis to $E$ faecium was 
mainly a shift to species with more intrinsic antibiotic resistance. Consequently this indicates that emerge of resistance is not due to mutations but selection of more resistant species. Most studies on the effect of antibiotic treatment on bowel flora have been performed in healthy humans. [13] Most of the antibiotics used for treatment in this study are mainly eliminated via the urinary tract, and the amount of antibiotics found in faeces is often low (approximately 1-5\%) [14-19]. An exception to this in our study is ertapenem where about $10 \%$ of the dose is found in the faeces [20]. This study wasn't powered to compare different treatment groups, effect on the faecal flora.

The strength of this study was that we investigated samples from patients treated for acute intra-abdominal infection in a clinical setting, thus hopefully reflecting clinical reality. There are few other studies where the whole potential pathogenic aerobic fecal flora has been examined after antibiotic therapy. In multicentre studies similar to ours, a substantial increase in bowel colonisation with resistant Enterobacteriaceae in patients treated with piperacillintazobactam or ceftriaxone in combination with metronidazol was shown, which is in agreement with our findings [8].

The practical choice of rectal swabs in favor of fecal samples may affect the amount of species that we were able to identify, due to the volume of the sample; this is a weakness of the study design. Since we used rectal swabs on all samples the differences we were able to show was actually differences in the flora. Another limitation of this study is that it was not designed or empowered to evaluate risk factors for acquiring antibiotic resistant Enterobacteriaceae with different antibiotic regiments. Furthermore, we did not analyse to see if there was a correlation between fecal colonisation and infection.

The antibiotic treatments applied were very disparate, due to the observational design, and it was not possible to evaluate each treatment strategy separately. This reflects the clinical settings diversity of antibiotic treatment and treatment length. A predetermined antibiotic 
treatment protocol with different treatment groups would make it possible to perform such an evaluation.

In our study we observed changes in the faecal flora and the emergence of Enterobacteriaceae with decreased susceptibility to cephalosporins and piperacillinetazobactam but not carbapenems, after antibiotic treatment. However, this study was not designed to determine if the changes in the faecal flora were caused by antibiotic treatment, the surgical intervention itself or cross transmission.

Pena et al has showed that carriage of Klebsiella pneumoniae-ESBL in the digestive tract is an independent variable associated with infection with the same $\mathrm{K}$ pneumoniae-ESBL, and the faecal carriage occurred 2-90 days prior of infection [21] Ben-Ami et al has shown that patients with ESBL-producing Enterobacteriaceae in faecal samples, at admission to a tertiary hospital in Tel Aviv, has grater risk of septicaemia with Ceftazidime-resistant isolate of the same species up to three month after admission (OR, 38.9;p<0,001). [22]These data suggests that Enterobacteriaceae in the rectal flora can cause infections in the host.

In conclusion, our results indicate that antibiotic treatment alters the distribution of species in the aerobic potential pathogenic faecal flora and increases the fraction of Enterobacteriaceae with decreased susceptibility to cephalosporins and piperacillintazobactam. These changes should be taken into consideration in patients with severe intraabdominal infections when initial treatment fails and further antibiotic treatment is required.

\section{References}


1. Donskey CJ. The role of the intestinal tract as a reservoir and source for transmission of nosocomial pathogens. Clin Infect Dis 2004;39:219-226

2. Guarner F, Malagelada J-R. Gut flora in health and disease. Lancet 2003;361:512-519

3. Backhed F, Ley RE, Sonnenburg JL, Peterson DA and Gordon JI. Host-bacterial mutualism in the human intestine. Science 2005;307:1915-1920

4. Hooper LV. Bacterial contributions to mammalian gut development. Trends Microbiol 2004;12:129-134

5. Solomkin JS, Mazuski JE, Bradley JS, et al. Diagnosis and management of complicated intra-abdominal infection in adults and children: guidelines by the Surgical Infection Society and the Infectious Diseases Society of America. Surg Infect 2010;11:79-109

6. Swenson BR, Metzger R, Hedrick TL, et al. Choosing Antibiotics for Intra-Abdominal Infections: What Do We Mean by High Risk? Surg Infect 2009;10:29-39

7. Loeffler JM, Garbino J, Lew D, Harbarth S and Rohner P. Antibiotic consumption, bacterial resistance and their correlation in a Swiss university hospital and its adult intensive care units. Scand J Infect Dis 2003;35:843-850

8. DiNubile MJ, Friedland I, Chan CY, et al. Bowel colonization with resistant gram-negative bacilli after antimicrobial therapy of intra-abdominal infections: observations from two randomized comparative clinical trials of ertapenem therapy. Eur J Clin Microbiol 2005;24:443-449

9. Aspevall O, Osterman B, Dittmer R, Stén L, Lindback E and Forsum U. Performance of four chromogenic urine culture media after one or two days of incubation compared with reference media. J Clin Microbiol 2002;40:1500

10. Murray PR, Baron EJ, Jorgensen JH, Pfaller MA and Landry ML. Manual of Clinical Microbiology. 9 ed. Vol. vol 1. Washington DC: American Society of Microbiology, 2007 11. Östholm-Balkhed $\AA$, Tärnberg $M$, Nilsson $M$, et al. Prevalence of extended-spectrum beta-lactamase-producing Enterobacteriaceae and trends in antibiotic consumption in a county of Sweden. Scand J Infect Dis 2010;42:831-838

12. Perez-Perez FJ, Hanson ND. Detection of Plasmid-Mediated AmpC \{beta\}-Lactamase Genes in Clinical Isolates by Using Multiplex PCR. J Clin Microbiol 2002;40:2153

13. Sullivan A, Edlund $C$ and Nord CE. Effect of antimicrobial agents on the ecological balance of human microflora. Lancet Infect Dis 2001;1:101-14

14. Harrison MP, Haworth SJ, Moss SR, Wilkinson DM and Featherstone A. The disposition and metabolic-fate of C-14 meropenem in man. Xenobiotica 1993;23:1311-1323

15. Mendelson J, Portnoy J and Sigman H. Pharmacology of gentamicin in the biliary tract of humans. Antimicrob Agents Chemother 1973;4:538

16. Rieder J. Excretion of sulfamethoxazole and trimethoprim into human bile. J Infect Dis 1973;128:Suppl: 574 p

17. Severn M, Powis SJA. Biliary-excretion and tissue-levels of cefuroxime-study in 11 patients undergoing cholecystectomy. J Antimicrob Chemother 1979;5:183-188

18. Sorgel F, Kinzig M. The chemistry, pharmacokinetics and tissue distribution of pipercillin tazobactame. J Antimicrob Chemother 1993;31:39-60

19. Soussy CJ, Deforges LP, Vanthoi JL, Feghali W and Duval JR. Cefotaxime concentration in the bile and wall of the gallbladder. J Antimicrob Chemother 1980;6:125-130

20. Wong BK, Xu X, Yu S, et al. Comparative disposition of [C-14]ertapenem, a novel carbapenem antibiotic, in rat, monkey and man. Xenobiotica 2004;34:379-389 
21. Pena C, Pujol M, Ardanuy $C$, et al. Epidemiology and successful control of a large outbreak due to Klebsiella pneumoniae producing extendedspectrum beta-lactamases. Antimicrob Agents Chemother 1998;42:53

22. Ben-Ami R, Schwaber MJ, Navon-Venezia S, et al. Influx of Extended-Spectrum BetaLactamase-Producing Enterobacteriaceae into the Hospital. Clin Infect Dis 2006;42:925 
Table I: Demographic data.

\begin{tabular}{|c|c|c|}
\hline & $\begin{array}{l}\text { Mean [SD] (Min- } \\
\text { Max) }\end{array}$ & Number of patients (\%) \\
\hline \multicolumn{3}{|l|}{ Age(years) } \\
\hline Women & 59 [18] (18-94) & $70(50)$ \\
\hline Men & 57 [20] (18-85) & $70(50)$ \\
\hline Total & $58[19](18-94)$ & 140 \\
\hline \multicolumn{3}{|l|}{ Unit } \\
\hline Norrköping & & $32(22,9)$ \\
\hline Västerås & & $31(22,1)$ \\
\hline Göteborg & & $30(21,4)$ \\
\hline Örebro & & $18(12,9)$ \\
\hline Helsingborg & & $11(7,9)$ \\
\hline Uppsala & & $9(6,4)$ \\
\hline Linköping & & $7(5,0)$ \\
\hline Karlstad & & $2(1,4)$ \\
\hline Total & & 140 \\
\hline \multicolumn{3}{|l|}{ Inclusion diagnosis } \\
\hline Appendicitis & & $49(35,0)$ \\
\hline Cholecystitis & & $28(20,0)$ \\
\hline Diverticulitis & & $23(16,4)$ \\
\hline Intra-abdominal abscess & & $23(16,4)$ \\
\hline Peritonitis & & $12(8,6)$ \\
\hline Perforation of the GIT & & $5(3,6)$ \\
\hline \multicolumn{3}{|l|}{ Surgical intervention } \\
\hline Appendectomy & & $44(31,4)$ \\
\hline Colectomy & & $13(9,3)$ \\
\hline Cholecystectomy & & $13(9,3)$ \\
\hline Drainage of abscess & & $5(3,6)$ \\
\hline Other & & $6(4,3)$ \\
\hline Explorative laparotomy & & $2(1,4)$ \\
\hline No surgical intervention & & $57(40,7)$ \\
\hline \multicolumn{3}{|l|}{ Discharge diagnosis } \\
\hline Appendicitis & & $51(36,4)$ \\
\hline Diverticulitis/diverticulosis & & $28(20,0)$ \\
\hline Cholecystitis/cholangitis/ & & $23(16,4)$ \\
\hline \multicolumn{3}{|l|}{ Cholelithiasis } \\
\hline Abdominal abscess & & $12(8,6)$ \\
\hline Other & & $26(18,7)$ \\
\hline Concomitant diagnosis & & $82(59)$ \\
\hline Hypertension & & $34(24)$ \\
\hline Diabetes & & $11(8)$ \\
\hline Malignancy & & $6(4)$ \\
\hline Asthma & & $6(4)$ \\
\hline Chronic arthritis & & $5(4)$ \\
\hline $\begin{array}{l}\text { Chronic obstructive pulmonary } \\
\text { disease }\end{array}$ & & $4(3)$ \\
\hline Previous antibiotic treatment $^{a}$ & & $27(19)$ \\
\hline
\end{tabular}


${ }^{\text {a }}$ Within 6 months preceding inclusion. 
Table II

Number and proportion of patients colonised with different species. Total number of patients $=140$.

\begin{tabular}{|c|c|c|c|c|}
\hline \multirow[t]{2}{*}{ Species } & \multicolumn{2}{|l|}{ Sample 1} & \multicolumn{2}{|l|}{ Sample 2} \\
\hline & $\begin{array}{l}\text { Number } \\
\text { of } \\
\text { patients } \\
\text { colonised }\end{array}$ & $\begin{array}{l}\text { Proportion } \\
\text { of patients } \\
\text { colonised } \\
(\%)\end{array}$ & $\begin{array}{l}\text { Number } \\
\text { of } \\
\text { patients } \\
\text { colonised }\end{array}$ & $\begin{array}{l}\text { Proportion of } \\
\text { patients } \\
\text { colonised } \\
(\%)\end{array}$ \\
\hline Escherichia coli & 126 & $(90.0)$ & 81 & (57.9) \\
\hline Enterococcus faecalis & 81 & (57.9) & 90 & (64.3) \\
\hline Yeast & 44 & (31.7) & 99 & (70.7) \\
\hline Klebsiella pneumoniae & 36 & (25.7) & 47 & (33.6) \\
\hline Enterococcus faecium & 30 & (21.4) & 70 & $(50.0)$ \\
\hline Citrobacter species & 23 & (16.4) & 38 & (27.1) \\
\hline Klebsiella oxytoca & 23 & (16.4) & 29 & (20.7) \\
\hline Enterobacter cloacae & 23 & (16.4) & 21 & $(15.0)$ \\
\hline Pseudomonas aeruginosa & 23 & (16.4) & 8 & (5.7) \\
\hline Pseudomonas species & 6 & (4.3) & 6 & (4.3) \\
\hline Enterobacteriaceae species $^{a}$ & 5 & (3.6) & 15 & (10.7) \\
\hline Morganella morganii & 5 & (3.6) & 1 & $(0.7)$ \\
\hline Acinetobacter species & 4 & (2.9) & 7 & (5.0) \\
\hline Stenotrophomonas maltophilia & 4 & (2.9) & 4 & (2.9) \\
\hline Proteus vulgaris & 3 & (2.1) & 6 & (4.3) \\
\hline $\begin{array}{l}\text { Enterococcus species non faecium, } \\
\text { non faecalis }\end{array}$ & 3 & (2.1) & 1 & $(0.7)$ \\
\hline Enterobacter agglomerans & 2 & (1.4) & 4 & (2.9) \\
\hline Proteus mirabilis & 2 & (1.4) & 3 & (2.1) \\
\hline Providentia species & 2 & (1.4) & 3 & (2.1) \\
\hline Serratia species & 1 & $(0.7)$ & 4 & (2.9) \\
\hline Gram-negative bacteriab $^{\mathrm{b}}$ & 1 & $(0.7)$ & 2 & (1.4) \\
\hline Kluyrvera species & 1 & $(0.7)$ & 1 & $(0.7)$ \\
\hline Vibiro vulnificus & 1 & $(0.7)$ & 0 & $(0.0)$ \\
\hline Hafnia alvei & 0 & $(0.0)$ & 2 & (1.4) \\
\hline Enterobacteriaceae species & 136 & (97.1) & 108 & $(77.1)$ \\
\hline $\begin{array}{l}\text { Pseudomonas species } \\
\text { /Acinetobacter species }\end{array}$ & 30 & $(21.4)$ & 21 & (15.0) \\
\hline
\end{tabular}

${ }^{a}$ Enterobacteriaceae species not further typed

${ }^{\mathrm{b}}$ Gram-negative bacteria not Enterobacteriaceae.

The gain or loss of a bacterial species was compared between Samples 1 and 2 using

McNemars test. The total number of species present was tested with pair-wise student's ttest. 
Table III. The difference mean fraction of Enterobacteriaceae (EBC) with decreased susceptibility and Pseudomonas species/ Acinetobacter species (NF), between the two

\begin{tabular}{|c|c|c|c|c|c|c|}
\hline \multirow[t]{2}{*}{ Antibiotic } & \multicolumn{3}{|c|}{ EBC } & \multicolumn{3}{|c|}{ NF } \\
\hline & $\begin{array}{l}\text { Sample } 1 \\
\text { Mean }^{\text {a }}\end{array}$ & $\begin{array}{l}\text { Sample } \\
2 \text { Mean } \\
a\end{array}$ & $p$ & $\begin{array}{l}\text { Sample } 1 \\
\text { Mean }^{b}\end{array}$ & $\begin{array}{l}\text { Sample } \\
2 \text { Mean }\end{array}$ & $\mathrm{p}$ \\
\hline Cefotaxime & 0.357 & 0.507 & 0.045 & & & \\
\hline $\begin{array}{l}\text { Ceftazidime } \\
\text { Cefuroxime }\end{array}$ & $\begin{array}{l}0.286 \\
0.600\end{array}$ & $\begin{array}{l}0.379 \\
0.800\end{array}$ & $\begin{array}{l}0.158 \\
0.032\end{array}$ & 0.043 & 0.043 & 1.000 \\
\hline Ciprofloxacin & 0.414 & 0.400 & 0.841 & 0.021 & 0.021 & 1.000 \\
\hline Gentamicin & 0.050 & 0.036 & 0.549 & & & \\
\hline Imipenem & 0.0 & 0.014 & 0.157 & 0.043 & 0.007 & 0.049 \\
\hline Piperacillin-Tazobactam & 0.307 & 0.450 & 0.041 & 0.014 & 0.021 & 0.640 \\
\hline $\begin{array}{l}\text { Trimethoprim- } \\
\text { sulphamethoxazole }\end{array}$ & 0.343 & 0.293 & 0.425 & & & \\
\hline
\end{tabular}

samples.

$\mathrm{p}<0,05$ considered significant

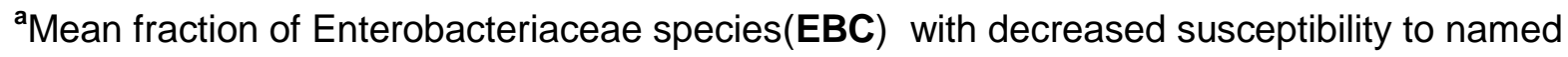
antibiotic per patient.

${ }^{\mathbf{b}}$ Mean fraction of Pseudomonas spp/ Acinteobact $\operatorname{spp}(\mathbf{N F})$ with decreased susceptibility to named antibiotic per patient.

The mean fractions of species with decreased susceptibility in the study population were compared between Samples 1 and 2 with two-way analysis of variance with time as fixed factor and patient as random factor. 


\section{Figure 1.}

Flowchart describing antibiotic treatment among the 140 patients studied. Bent arrows indicate end of treatment. Number in parenthesis indicates number of patients, receiving mentioned antibiotic.






\section{Figure 2.}

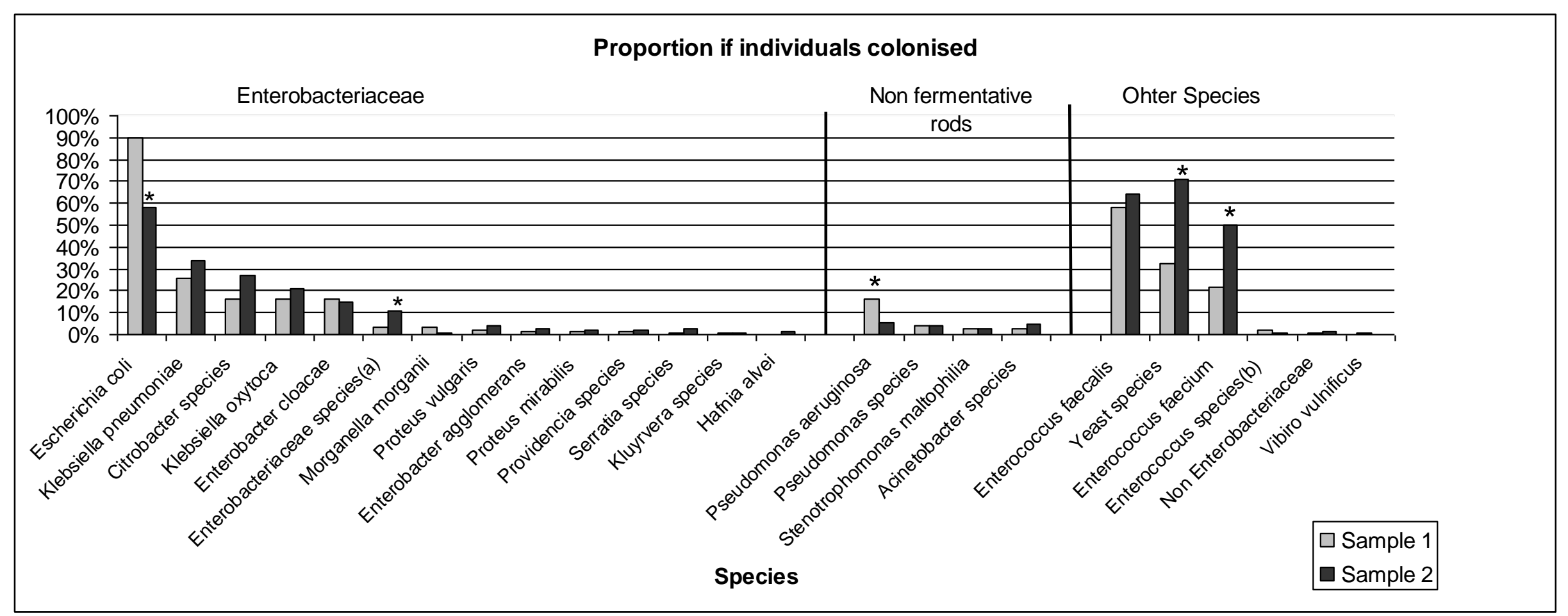

* Indicates significant differences between Sample 1 and Sample 2.

Sample 1: rectal sample obtained on admission

Sample 2: rectal sample obtained 2-14 days after the end of antibiotic treatment.

${ }^{a}$ Enterobacteriaceae species: glucose fermenting aerobic gram-negative bacteria where standard biochemical methods were not conclusive. 
${ }^{\mathrm{b}}$ Enterococcus species not classified as Enterococcus faecalis or Enterococcus faecium.

The gain or loss of a bacterial species was compared between Samples 1 and 2 using McNemars test. The total number of species present was tested with pair-wise student's t-test. 
